\title{
Performance Analysis of High Speed Optical Communication using FBG
}

\author{
Ashish Kumar Pandey \\ PG Student \\ Department of Electronics and \\ Communication Engineering \\ Shiats, Allahabad
}

\author{
Neelesh Agrawal \\ Assistant Professor \\ Department of Electronics and \\ Communication Engineering \\ Shiats, Allahabad
}

\author{
A. Ashok \\ Assistant Professor \\ epartment of Electronics and \\ Communication Engineering \\ Shiats, Allahabad
}

\begin{abstract}
This paper based on study of communication system over fiber optics. To design high speed system with high performance it is necessary to compensate the dispersion with the help of Fiber Bragg Grating. The performance of communication system simulation is done on Optisystem simulator Vr.12 at different parameters of the system. A high speed NRZ signal is transmitted over SSMF. By simulating the design of optical communication system at different values which includes Q-Factor, Min BER, MZM-Extinction Ratio, FBG length is evaluated. With the help of eye diagram get better result.
\end{abstract}

\section{Keywords}

Optical Fiber Communication, Fiber Bragg Grating , Dispersion compensation, Optisystem Simulator .

\section{INTRODUCTION}

Optical communication is way of information transmitting from transmitter to receiver with the help of light as mode and optical fiber as a medium. There are three main elements of optical communication system, light source at transmitter side (convert electrical information in the form of light), transmission medium (optical fiber) and light detector at receiver side (converts optical signal into electrical signal). FBG is an important element in optical fiber communication as dispersion compensator, gain flattener and filter. FBG is used with optical fiber to improve higher compression ratio. Chromatic and polar mode dispersion takes place in single mode fiber. In optical communication EDFA (erbium doped fiber amplifier) is used to compensate dispersion. Chromatic dispersion is dependent on wavelength. Optisystem simulator is innovative, advanced, easy, powerful simulator to design , test and evaluate broad area of spectrum almost any type of optical communication system for LAN(local area network) and MAN(metropolitan area network). Optisystem also offers optical communication system design and planning of component to system level. It is very helpful for users to design, analyze and simulate in various application such as WDM /TDM, IPTV, CATV network, amplifier, transmitter , RoF design and others. Optisystem is software which does not depend upon different simulation design. It is based upon real fiber optics modeling in optical communication system. Optisystem component library contains various official components.

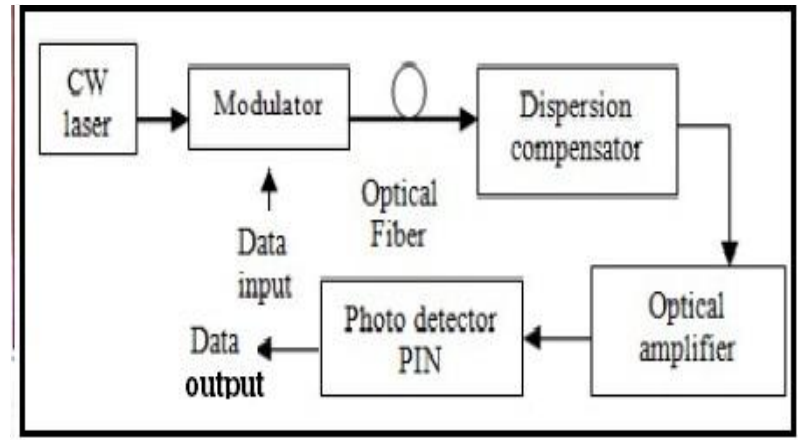

Figure 1.Block diagram of optical communication system.

In this paper simulation on optical communication system is done and analyzed the effect of component on receiver output by varying different parameter of communication system. At the receiver Q-factor is evaluated.

\section{FIBER BRAGG GRATING}

FBG (fiber bragg grating) is simple and cost effective filter for selective wavelength which is essential for low cost and high efficient optical network. FBG has quality of filtering and reflecting as well as highly efficient and low noises.

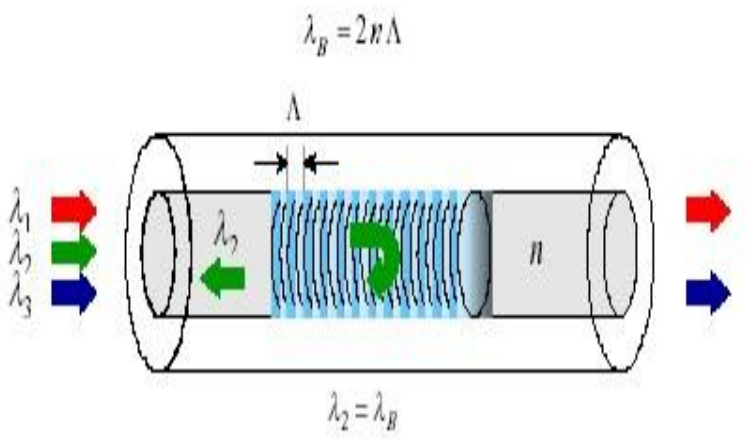

Figure 2. Basic Principle of FBG.

In optical fiber communication system chromatic dispersion is minimized with the help of FBG. FBG shows an expected effect on result of communication design by compensating chromatic dispersion. FBG in single mode fiber is directly applied to core of the fiber for periodic pattern of intense UV light. Refractive index of fiber is permanently increased by direct exposure. This will create fixed index modulation called as grating. 


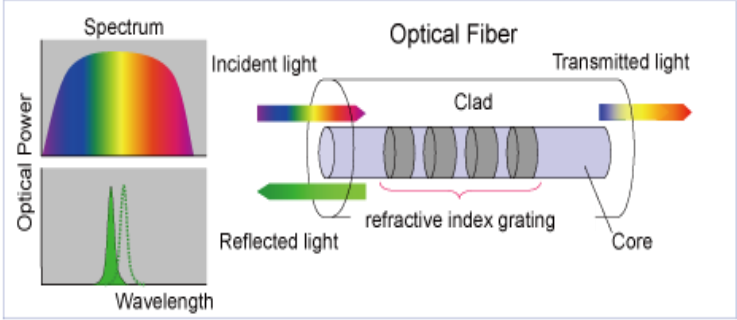

Figure 3. Operation of FBG.

Small reflected light is generated when periodic refraction is applied. Several small reflected light is combined to build a larger one at a fixed wavelength .The wavelength is called as Bragg's wavelength, which is approximately equals to half of the incident light wavelength. The remaining light (other than bragg's light) is transparent. The relation between Braggs wavelength, effective refractive index and grating periodicity is given by :

$$
\lambda B=2 \mathrm{n} \Lambda
$$

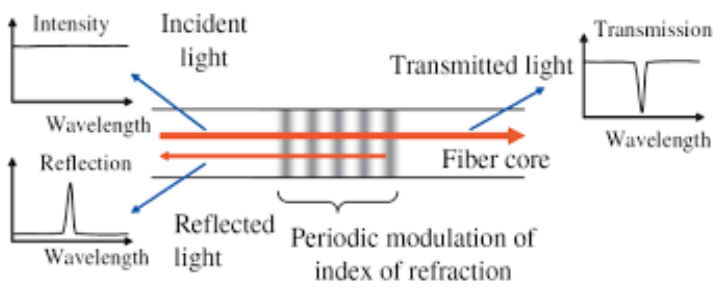

Figure 4. Working of FBG.

When a light transmitted through optical fiber light get dispersed. Broaden of pulse width due to higher wavelength lags over shorter wavelength. That's why at sufficiently high speed and at longer fiber length signals are start to overlap each other . So the maximum data transmitted through the fiber gets limited in this way. The dispersed pulse data is applied on chirped fiber bragg grating.The longer wavelength get reflected early but shorter wavelength get relatively delayed as compare to longer one .The grating is designed in such a way that all wavelengths exit the reflector at same instant . Due to which dispersion of the fiber pulse gets neutralize.

\section{DESCRIPTION OF COMPONENT}

NRZ (non return to zero) pulse generator having ability to control bandwidth. It helps the data bit to convert in electrical signal for modulation purpose. PRBS (pseudo random bit sequence ) is used to produce scramble data in terms of bps . MZM (mach-zehnder modulator) is used based on the principle of interferrometry. It has two inputs one for data and other one as optical carrier and also a output for modulated optical signal at a fixed extinction ratio. CW (continuous wave) laser is used to generate high frequency optical carrier signal. SMF (single mode fiber) is used for high speed and long distance communication. FBG (fiber bragg grating) is used to compensate chromatic dispersion in optical fiber. EDFA (erbium doped fiber amplifier) to boost up the optical signal get attenuated over the fiber. At receiver PIN diode is used as a photo detector to covert optical transmitted signal to electrical signal.

\section{DESIGN CONSIDERATION}

The basic components of optical communication system are transmitter, communication channel and receiver. The transmitter transmits information to the receiver with the help of optical fiber. PRBS transmits information in the form of bit 0 or 1 at higher rate. NRZ pulse generator convert bit form of information to electrical signal. MZM modulates electrical information signal with optical carrier signal produced by $\mathrm{CW}$ laser. Optical modulated signal is transmitted through SMF. EDFA is used at transmitter and receiver end. FBG is used after EDFA to compensate dispersion. Optical signal is converted into electrical form with the help of PIN diode. At receiver side signal is visualized by BER analyzer and Eye diagram analyzer.

\section{Table 1. Simulation Parameters}

\begin{tabular}{|l|l|}
\hline PRBS DATA RATE & $20 \mathrm{Gbps}$ \\
\hline CW LASER INPUT POWER & $5 \mathrm{db}$ \\
\hline CW LASER FREQUENCY & $193.1 \mathrm{Thz}$ \\
\hline REFERENCE WAVELENGTH & $1550 \mathrm{~nm}$ \\
\hline FIBER LENGTH & $10 \mathrm{KM}$ \\
\hline ATTENUATION AT CABLE SECTION & $0.2 \mathrm{db} / \mathrm{KM}$ \\
\hline EDFA LENGTH & $5 \mathrm{~m}$ \\
\hline
\end{tabular}

\section{Case-I}

Simulation done at different extinction ratio of Mach-Zehnder modulator. The extinction ratio is varied from $10 \mathrm{db}$ to $50 \mathrm{db}$ at each $10 \mathrm{db}$ variation, by test and try method. At FBG length $6 \mathrm{~mm}$.The simulated result is evaluated in the form table of the output simulated results.

\section{Case-II}

Simulation done at different grating length of FBG . The grating length is varied from $1 \mathrm{~mm}$ to $9 \mathrm{~mm}$ at each $1 \mathrm{~mm}$ variation, by test and try method. At $7 \mathrm{db}$ extinction ratio. The result is evaluated in the form table of output simulated results.

\section{SIMULATION DESIGN}

The simulation design of optical communication system is designed with the help of optisystem version 12 . The design of the system is given in figure 5 . 


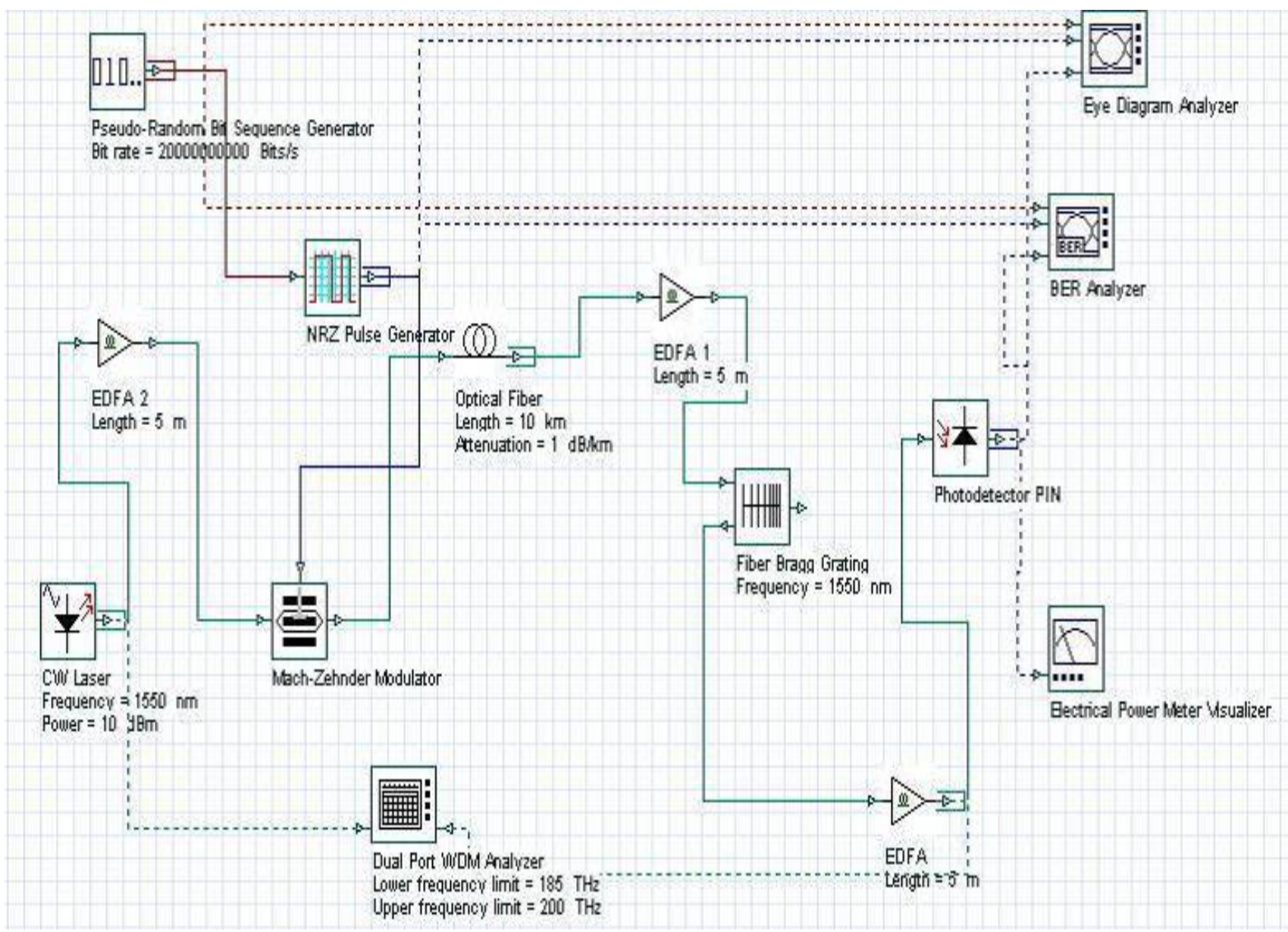

Figure 5. Simulation design of optical high speed communication system .

\section{RESULTS \& DISCUSSION}

Simulated system design on optisystem simulation software. Eye diagram are shown and results are tabulated in tables.

Case-I - Simulation at different extinction ratio .

Table 2. The output reading of simulation is tabulated for different extinction ratio of $\mathrm{MZ}$ modulator .

\begin{tabular}{|c|c|l|}
\hline MZ modulator extinction ratio & Max Q-factor & Min BER \\
\hline $10 \mathrm{db}$ & 25.38 & $1.87 * \mathrm{e}-142$ \\
\hline $20 \mathrm{db}$ & 21.24 & $1.49 * \mathrm{e}-100$ \\
\hline $30 \mathrm{db}$ & 20.28 & $7.81 * \mathrm{e}-92$ \\
\hline $40 \mathrm{db}$ & 19.22 & $9.89 * \mathrm{e}-83$ \\
\hline $50 \mathrm{db}$ & 18.59 & $1.3 * \mathrm{e}-77$ \\
\hline
\end{tabular}

Case-II - Simulation at different FBG length .

Table 3. The output reading of simulation is tabulated for different FBG length .

\begin{tabular}{|c|c|l|}
\hline FBG length & Max Q-factor & \multicolumn{1}{|c|}{ Min BER } \\
\hline $1 \mathrm{~mm}$ & 7.44 & $3.84 * \mathrm{e}-14$ \\
\hline $2 \mathrm{~mm}$ & 8.06 & $3.12 * \mathrm{e}-16$ \\
\hline $3 \mathrm{~mm}$ & 10.99 & $1.78 * \mathrm{e}-28$ \\
\hline $4 \mathrm{~mm}$ & 27.34 & $5.6 * \mathrm{e}-165$ \\
\hline $5 \mathrm{~mm}$ & 25.24 & $5.51 * \mathrm{e}-141$ \\
\hline $6 \mathrm{~mm}$ & 24.51 & $5.19 * \mathrm{e}-133$ \\
\hline $7 \mathrm{~mm}$ & 16.20 & $2.32 * \mathrm{e}-59$ \\
\hline $8 \mathrm{~mm}$ & 10.41 & $1 * \mathrm{e}-25$ \\
\hline $9 \mathrm{~mm}$ & 7.43 & $5.1 * \mathrm{e}-14$ \\
\hline
\end{tabular}

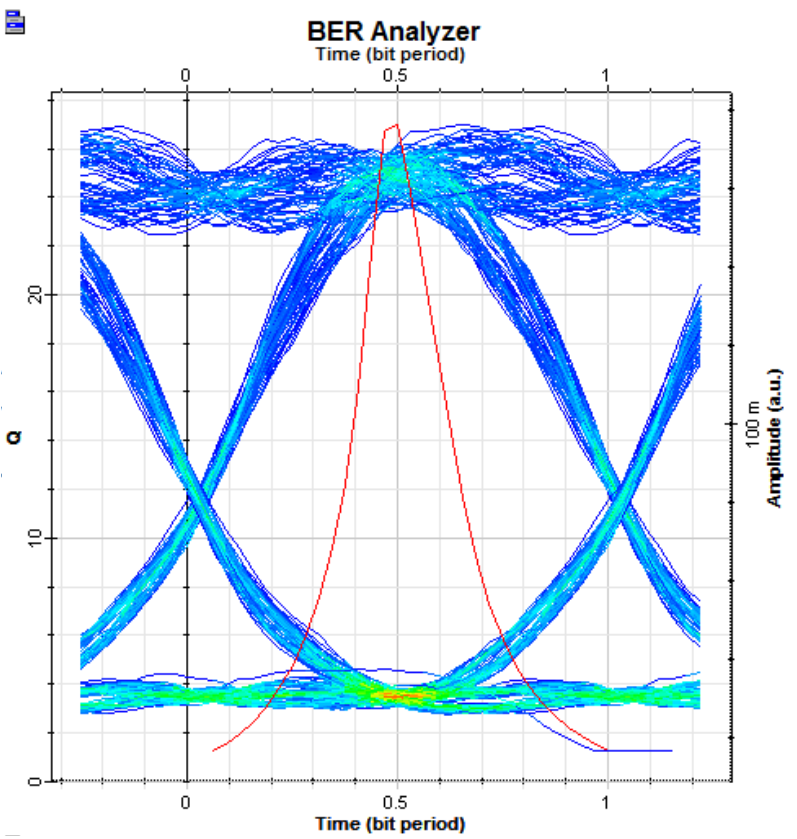

Figure 6. Eye diagram of simulated design at 7db extinction ratio at $4 \mathrm{~mm}$ fbg length .

\section{CONCLUSION}

From the simulation and design of optical communication system studied . In case-I Q-factor of the system is gradually decreasing with increase in extinction ratio of $\mathrm{MZ}$ modulator. 
In case-II initially Q-factor is increasing till $4 \mathrm{~mm}$ and at $4 \mathrm{~mm}$ FBG length it is maximum and after $4 \mathrm{~mm}$ the value is gradually decreasing. This concludes that at $4 \mathrm{~mm}$ FBG length is perfect for the design and also Q-factor is inversely vary with extinction ratio.

This conclusion is useful for design and implementation of future optical communication system and network

\section{REFERENCES}

[1] A.Bharadwaj , G.Soni. 2015, “Performance Analysis of 20Gbps Optical Transmission System Using Fiber Bragg Grating , A.C.E.T.

[2] S.Kumar, A.K.Jaiswal , M.Kumar, R.Saxena 2013 "Performance Analysis of Dispersion Compensation in Long Haul Optical Fiber with DCF ,Shiats .

[3] Othman, Sulaiman , Ismail , Mishran ,Said ,Rahim , Chee Pee ,Motsidi , 2012 "An Analysis of 10 Gbits/s Optical Transmission System using Fiber Bragg Grating (FBG), Malaysia.

[4] M.Kaur , H.Sarangal , 2015 "Simulation of Optical Transmission System to Compensate Dispersion Using
Chirped Fiber Bragg Grating

[5] A.Bharadwaj, G.Soni. 2015 "Performance Analysis of Optical Communication System Using Fiber Bragg Grating.

[6] B.Prasad, B.Mallick ,A.K.Parida, 2014. "Fiber Bragg Grating as a Dispersion Compensator in an Optical Transmission System Using Optisystem Software.

[7] N.Salehi , S.Mohmmadi ,M .Salehi ,M.Zahidi , 2014 “ Simulation of a transmission system for comparison two compensator with optisystem software.

[8] K.Kumar , A.K.Jaiswal , M.Kumar , N.Agrawal , 2014 “ Performance Analysis of dispersion compensation using Fiber Bragg Grating (FBG) in Optical Communication .

[9] A.J.Agrawal ,A.K.Jaiswal , M.Kumar, R.Saxena "Analysis to Compensate Dispersion in Optical Communication Link Using Chirp Grating . 